\title{
Reducing the parameter space for Unparticle-inspired models using white dwarf masses
}

\author{
Rodrigo Alvares de Souza ${ }^{*}$ and J.E. Horvath \\ Instituto de Astronomia, Geofísica e Ciências Atmosféricas da USP \\ Rua do Matão 1226, Cidade Universitária, 05508-090, São Paulo, Brazil
}

(Dated: July 1, 2018)

\begin{abstract}
Based on astrophysical constraints derived from Chandrasekhar's mass limit for white-dwarfs, we study the effects of the model on the parameters of unparticle-inspired gravity, on scales $\Lambda_{U}>1 \mathrm{TeV}$ and $d_{U} \approx 1$.
\end{abstract}

PACS numbers: 04.20.Fy, 04.80.Cc, 04.25.Nx

\section{INTRODUCTION}

Many proposals for explaining the apparent shortcomings of the Standard Model have been advanced. The Unparticle Model proposed by Georgi [1] aimed to include in the Standard Model massive but scale-invariant particles, sharing the same physics of the scale-dependent counterparts. These objects, called 'unparticles', could play an important role in low-energy physics [2], since the model implies that unparticles can be exchanged between massive particles, leading to a new force called 'ungravity'. This "fifth" force would add a perturbation term to the newtonian gravitational potential, although the exact potential can not be obtained because the distance at which the perturbed potential matches the newtonian expression needs to be known. In order to bypass this limitation, the perturbed potential has been assumed to be of the form [2]

$$
V(r)=-\frac{G M}{2 r}\left[1+\left(\frac{R_{G}}{r}\right)^{2 d_{U}-2}\right],
$$

where $d_{U}$ (the scaling dimension of the unparticles operator $\left.O_{U}\right)$ is $\approx 1$, as a reasonable approximation, and $R_{G}$ is the characteristic length scale of ungravity, given by

$$
\begin{aligned}
R_{G} & =\frac{1}{\pi \Lambda_{U}}\left(\frac{M_{P l}}{M_{*}}\right)^{1 /\left(d_{U}-1\right)} \times \\
& \times\left[\frac{2(2-\alpha)}{\pi} \frac{\Gamma\left(d_{U}+1 / 2\right) \Gamma\left(d_{U}-1 / 2\right)}{\Gamma\left(2 d_{U}\right)}\right]^{1 /\left(d_{U}-1\right)}
\end{aligned}
$$

where $\Lambda_{U}$ is the energy scale of the unparticle interactions, $M_{P l}=2.4 \times 10^{18} \mathrm{GeV}$ is the Planck mass and $\alpha$ is a constant dependent on the type of propagator considered.

The problem addressed in this work is to determine the bounds of the mass of the interaction (un)particle

\footnotetext{
*Email: rodrigo.souza@usp.br

†Email: foton@astro.iag.usp.br
}

$M_{*}$ with $d_{U} \simeq 1$. For this purpose, a suitable quasinewtonian gravitational system needs to be studied and compared with the pure newtonian results. A first study of this regime by Bertolami, Páramos and Santos [3] has addressed the stellar equilibrium problem, deriving a perturbed Lane-Emden equation further applied to the Sun. They explored the well-known similarity of the full stellar structure to an $n=3$ polytropic model, and derived limits from the maximum allowed uncertainty in the central temperature $\Delta T_{c} / T_{c}=0.06$.

In spite of the successful derivation of meaningful limits to the unparticle parameters, it is known that the detailed structure of the Sun is actually quite complicated, and many physical factors have to be considered beyond the simplest Chandrasekhar's polytropic model 4]. Therefore, it is worth considering another very wellknown system to which the Chandrasekhar theory gives an even better representation: the white dwarf sequence. We shall show below that an important feature of these sequences (the maximum mass) is sensitive to the unparticle quantities and allows to impose strong limits on them.

\section{STELLAR EQUILIBRIUM AND WHITE-DWARFS}

Since Chandrasekhar's polytropic model is widely known, we briefly recall how the unparticle theory modifies it, as first shown by Bertolami, Páramos and Santos 3]. The equations of stellar hydrostatic equilibrium and mass conservation can be reduced to a second order differential equation, if a polytropic equation of state of the form $P=K \rho^{1+1 / n}$ is assumed to hold. If the density is written as $\rho=\rho_{c} \theta^{n}$, and the radius as $r=\beta \xi$, one can easily obtain the original Lane-Emden equation

$$
\frac{1}{\xi^{2}} \frac{d}{d \xi}\left(\xi^{2} \frac{d \theta}{d \xi}\right)=-\theta^{n}
$$

where $n$ is the polytropic index, $\xi$ is the dimensionless radius and $\beta$ is given by 


$$
\beta=\left[\frac{(n+1) K}{4 \pi G} \rho_{c}^{(1 / n)-1}\right]^{\frac{1}{2}},
$$

$K$ being the polytropic constant dependent on the specific value of $n$. This equation is subject to the usual boundary conditions: $\rho(r=0)=\rho_{c}$ and $d P / d r=0$ for $r=0$, which translates to $\theta(\xi=0)=1$ and $\theta^{\prime}(\xi=0) \equiv d \theta / d \xi=0$. The detailed derivation of the Lane-Emden equation can be consulted in the classical reference [4]. These results can be used to derive the mass-radius relation, given by Eq.(51).

$$
m(r)=4 \pi\left(\frac{r}{\xi_{*}}\right)^{\frac{3-n}{1-n}}\left(\frac{(n+1) K}{4 \pi G}\right)^{\frac{n}{n-1}} \xi_{*}^{2}\left|\theta^{\prime}\left(\xi_{*}\right)\right|
$$

Considering that white-dwarfs are small stars composed by electron-degenerate matter, in which the core material no longer undergoes fusion reactions, the LaneEmden equation describes very well their behavior as a result of the proximity of the electronic component to a polytropic form. As is well-known, in the non-relativistic limit the white dwarf matter can be represented by a $n=3 / 2$ polytrope and in the relativistic case the $n=3$ is quite accurate.

The same technique employed by Chandrasekhar can be used to obtain the perturbed Lane-Emden equation. From the perturbed potential given by Eq.(1), the perturbed gravitational acceleration can be obtained via $\vec{F}=-\vec{\nabla} V[\underline{5}]$, used to determine the hydrostatic equilibrium equation, as seen in Eq.(6).

$$
\frac{d P}{d r}=-\frac{G M \rho}{2 r^{2}}\left[1+\left(2 d_{U}-1\right)\left(\frac{R_{G}}{r}\right)^{2 d_{U}-2}\right]
$$

Then, from this hydrostatic equilibrium equation, Bertolami, Páramos and Santos derived a perturbed Lane-Emden equation

$$
\begin{aligned}
\frac{1}{\xi^{2}} & \frac{d}{d \xi}\left(\xi^{2} \frac{d \theta}{d \xi}\right)= \\
& \frac{-\theta^{n}}{2}\left[1+\left(2 d_{U}-1\right)\left(\frac{\xi_{G}}{\xi}\right)^{2 d_{U}-2}\right]- \\
& -\left(2 d_{U}-1\right)\left(d_{U}-1\right) \frac{1}{\xi} \frac{d \theta}{d \xi}\left(\frac{\xi_{G}}{\xi}\right)^{2 d_{U}-2}
\end{aligned}
$$

in order to examine meaningful bounds on the parameters $R_{G}$ in Eq.(2), from which one can obtain the bounds of the mass of the interaction (un)particle $M_{*}$ based on astrophysical constraints (the parameter $\xi_{G}=R_{G} / \beta$ has been defined here to simplify the expressions). We have corrected a slight misprint in the paper [3] which would preclude the derivation of Eq.(17), related to the definition of the variable $\beta$ as given by (4).
Once these matters are settled, the perturbed massradius relation can be obtained in the usual manner, being formally identical to the analogous mass-radius relation given by Chandrasekhar (Eq.(5)), but with the perturbative effects present through the solutions of the perturbed Lane-Emden equation and its derivative $\left(\theta\left(\xi_{1}\right)\right.$ and $\theta^{\prime}\left(\xi_{1}\right)$ ), respectively, both evaluated at the first zero as in Ref.[4]. This modification sets the stage for an analysis leading to novel bounds on the unparticle parameters. Since we are interested on the maximum possible mass for a white dwarf, we set the parameters related to the Lane-Emden model to the relativistic limit (polytropic index $n=3$ ), and the white dwarf mass-radius relation becomes

$$
M=4 \pi\left(\frac{K}{\pi G}\right)^{\frac{3}{2}} \xi_{*}^{2}\left|\theta^{\prime}\left(\xi_{*}\right)\right|
$$

losing any dependence on the radius, as expected.

The key feature pointed out above, related to the explicit dependence of the maximum mass through the zero of the Lane-Emden function and its slope, leads to quite a strong dependence of the maximum mass with $d_{U}$ and $R_{G}$, which can be substantially different from the "canonical" $M_{C h}=1.457\left(2 / \mu_{e}\right)^{2} M_{\odot}[4,6]$.

\section{OBSERVATIONS AND ANALYSIS}

Following the framework presented by Bertolami, Páramos and Santos, we obtained the numerical solutions into Eq.(7) varying both parameters $\xi_{G}$ and $d_{U}$, and used these solutions on Eq. (8) to determine the masses associated to the combination of $d_{U}$ and $\xi_{G}$. It is important to note that the combination $\xi_{G}$ and $d_{U}$ generated a broad range of masses, spanning from $M \approx 1.291 M_{\odot}$ to $M \approx 1.874 M_{\odot}$. This in turn means that it is possible to constrain the parameters based on the maximum mass for white dwarfs, because this mass must complain with the values obtained by observational data. Following this reasoning, we assumed three reasonable values for the maximum mass

- the maximum observed masses in a large white dwarf sample, from the recent work by Kepler and collaborators [7], is $M=1.33 M_{\odot}$. Nevertheless, in order to effectively constrain the values of $d_{U}$ near 1 , we had to consider masses of at least $M=1.36 M_{\odot}$, otherwise $d_{U}$ would be far from 1 and the gravitational corrections in Eq.(1) would be too large;

- the "canonical" limit, $M_{C h}=1.457 M_{\odot}$ for carbontype white dwarfs; in spite of this widely accepted value is still beyond the actual observed maximum, its use seems very reasonable 
- an even larger value, above the former "Chandrasekhar's" limit, $M=1.60 M_{\odot}$, arbitrarily chosen to represent an extreme limit allowed in nature. If true, the number of objects between the actually observed maximum $M=1.33 M_{\odot}$ and this proposed extreme value of $M=1.60 M_{\odot}$ must be substantial in a large sample such as the one analyzed by Kepler et al. 7], although none has been actually reported.

From the data above mentioned, we constructed the contour plots where the combination of $d_{U}$ and $\xi_{G}$ generates the desired maximum mass. This plots are depicted in Fig 1 for ranges larger than the radius of the star $\left(\xi_{G}>1, d_{U} \lesssim 1\right)$ and in Fig 2 for ranges smaller the radius of the $\operatorname{star}\left(\xi_{G}<1, d_{U} \gtrsim 1\right)$. One should note that for $d_{U} \gtrsim 1$, only masses of $M=1.36 M_{\odot}$ and $M=1.457 M_{\odot}$ are obtained, while a mass of $M=1.6 M_{\odot}$ can be imposed for $d_{U} \lesssim 1$ only.

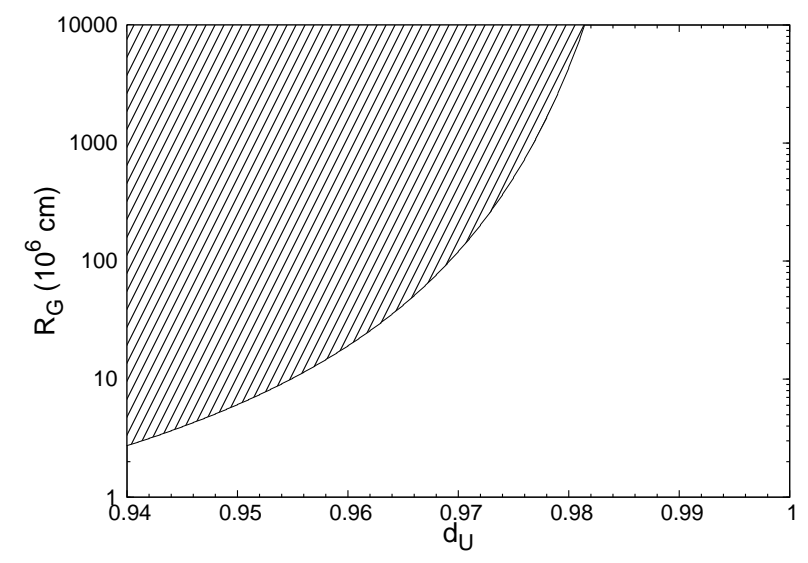

FIG. 1: Contour plot (locus) of maximum mass $(M=$ 1.6 $M_{\odot}$ ) in the $R_{G}-d_{U}$ plane, for an interaction range larger than the radius of the star. The dashed area gives masses bigger than $M=1.6 M_{\odot}$, and should be considered as forbidden.

The connection with the (un)particle mass follows by solving equation Eq.(2), for the ratio $M_{*} / M_{P l}$, namely

$$
\begin{aligned}
\frac{M_{*}}{M_{P l}} & =\left[\pi \Lambda_{U} R_{G}\left(d_{U}\right)\right]^{1-d_{U}} \times \\
& \times\left[\frac{2(2-\alpha)}{\pi} \frac{\Gamma\left(d_{U}+1 / 2\right) \Gamma\left(d_{U}-1 / 2\right)}{\Gamma\left(2 d_{U}\right)}\right]^{1 / 2},
\end{aligned}
$$

which, by using $R_{G}\left(d_{U}\right)$ defined in the plots above, it is possible to infer the mass of the interaction (un)particle $M_{*}$. We point out that this procedure differs significantly from the method used by Bertolami, Páramos and Santos [3], where the $6 \%$ uncertainty leads to terms $R_{-}\left(d_{U}\right)$ and $R_{+}\left(d_{U}\right)$, because we use exact values for the maximum masses. Nevertheless, it is clear that the ratio must be interpreted as a lower bound to the ratio $M_{*} / M_{P l}$, considering that lower maximum masses (which would be in

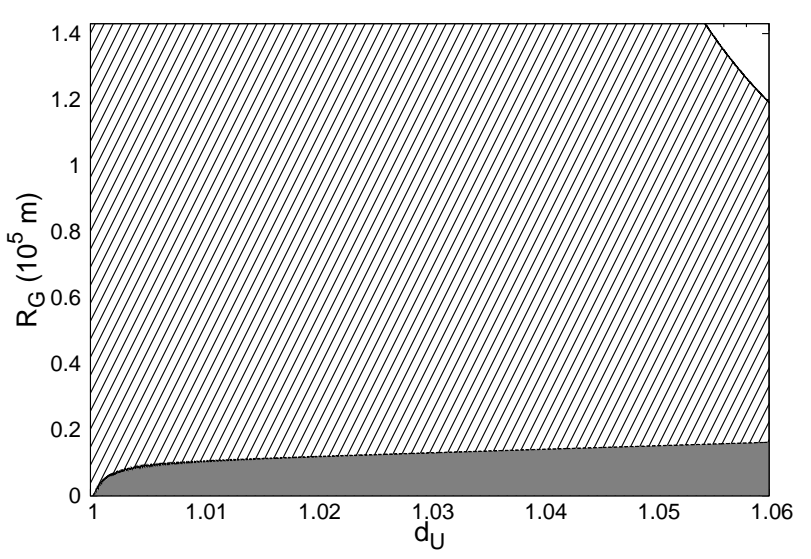

FIG. 2: Contour plot (locus) of maximum masses $M=$ $1.36 M_{\odot}$ and $M=1.457 M_{\odot}$ in the $R_{G^{-}} d_{U}$, for an interaction range smaller than the radius of the star. The curve spanning from $1.055<d_{U}<1.06$ (upper right corner of the graphic) produces a maximum mass of $M=1.36 M_{\odot}$ and the curve between $1<d_{U}<1.06$ (lower side of the graphic) the mass of $M=1.457 M_{\odot}$. The upper white area gives maximum masses lower than $M=1.36 M_{\odot}$, the dashed area yields masses in the range $1.36 M_{\odot}<M<1.457 M_{\odot}$, and the lower area produces masses bigger than $1.457 M_{\odot}$. If one accepts the latter value as the maximum allowed, the dark area is forbidden

conflict with observations, as stated before) would result in even lower ratios. Plotting the ratio as a function of $d_{U}$ and fixing the parameters $\alpha$ and $\Lambda_{U}$ in the same way as Bertolami, Páramos and Santos, we obtain the ranges for the mass of the interaction (un)particle depicted in Fig 3 and Fig 4 for the case of the "canonical" mass and two different choices of $\Lambda_{U}$.

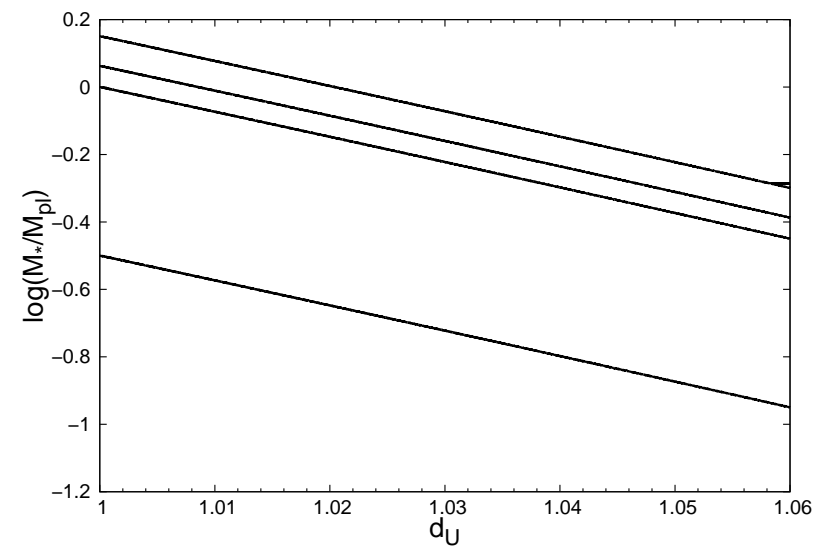

FIG. 3: Lower bounds on $\log \left(M_{*} / M_{p l}\right)$, for $\alpha=0, \alpha=2 / 3$, $\alpha=1, \alpha=1.9$, from top to bottom respectively. The fixed values are $\Lambda_{U}=1 \mathrm{TeV}$ and $M=1.457 M_{\odot}$.

Analyzing the data for $M=1.36 M_{\odot}, d_{U}$ s fall in the range $1.055<d_{U}<1.060$. Therefore, this maximum mass yields a lower bound to $M_{*}$ in the range $(0.03-$ $0.50) M_{p l}$. For $M=1.457 M_{\odot}, d_{U}$ s lies in the range 


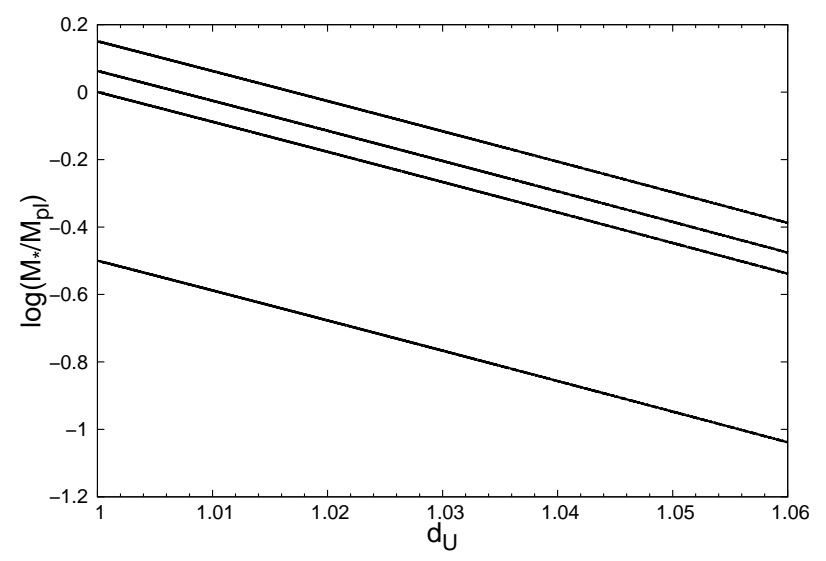

FIG. 4: Lower bounds on $\log \left(M_{*} / M_{p l}\right)$, for $\alpha=0, \alpha=2 / 3$, $\alpha=1, \alpha=1.9$ from top to bottom respectively. The fixed values are $\Lambda_{U}=10^{3} \mathrm{TeV}$ and $M=1.457 M_{\odot}$.

$1<d_{U}<1.06$, yielding a lower bound to $M_{*}$ in the range $(0.1-1.6) M_{p l}$. The last case, with an assumed maximum mass $M=1.6 M_{\odot}$ and the interaction range larger than the star radius, the only allowed values for $d_{U}$ s are in the range $0.94<d_{U}<0.982$, and lower bounds to $M_{*}$ in the range $(0.5-6.3) M_{p l}$.

\section{CONCLUSIONS}

We have shown in this work that quite strong limits to the unparticle parameters can be obtained by using a simple form of the polytropic theory of Chandrasekhar adding a perturbation to the Lane-Emden equation, as first obtained by Bertolami, Páramos and Santos [3], and applying it to the white dwarf sequences.
The key point elaborated here is that a change on the unparticle parameters would affect the maximum mass allowed to white dwarfs, and thereby we explored this characteristic in order to limit the values of such parameters.

The requirement that the maximum mass can not be too small (because it would conflict with a few massive stars [7] or too big (because it would lead to unobserved supermassive white dwarfs) limit the values of $M_{*}$ to a confidence range of $0.1 M_{P l}<M_{*}<1.6 M_{P l}$ from this analysis alone for the case $d_{U} \gtrsim 1$. For the case $d_{U} \lesssim 1$, the mass-radius relation gives only masses bigger than the canonical value. Considering that until today there is no observation of white dwarfs with such high masses, this analysis may be interpreted to mean that values of $d_{U}<1$ are not allowed.

Following a different approach, based on a cosmological scenario, Bertolami and Santos [13] considered the variation of the gravitational coupling at the time of big bang nucleosynthesis, tensor exchange and the scaling dimension $d_{U}=1.1$ and found $M_{*}$ to be $>0.05 M_{P l}$, which is very close to the bounds found for $1<d_{U}<1.06$. Other works studying complementary bounds [3, 8, 12 ] could be combined to address the viability of a general unparticle model, unless one can manage to evade the bounds altogether. Even if so, a general argument to constrain the admissible perturbations to the newtonian potential can be made via the perturbed Lane-Emden equation, resorting to the observed massive white dwarfs.

\section{Acknowledgments}

We wish to acknowledge the support of the CNPq and FAPESP Agencies for financial support.
[1] H. Georgi, Phys. Rev. Lett. 98 (2007), URL arXiv:hep-ph/0703260v3

[2] H. Goldberg and P. Nath, Phys. Rev. Lett. 100, 031803 (2008).

[3] O. Bertolami, J. Páramos, and P. Santos, Phys. Rev. D 80, 022001 (2009).

[4] S. Chandrasekhar, Introduction to the Study of Stellar Structure (Dover, 1967), 3rd ed.

[5] D. A. Ostlie and B. W. Carrol, Modern Stellar Astrophysics (Addison-Wesley, 1996).

[6] L. Landau, Collected papers of L.D. Landau (Pergamon Press, 1965).

[7] S. O. Kepler, S. J. Kleinman, A. Nitta, D. Koester, B. G. Castanheira, O. Giovannini, A. F. M. Costa, and L. Al- thaus, 1315 (2007), arXiv:astro-ph/0612277.

[8] H. Davoudiasl, Phys. Rev. Lett. 99, 141301 (2007).

[9] A. Freitas and D. Wyler, Journal of High Energy Physics 2007, 033 (2007).

[10] N. Deshpande, S. D. Hsu, and J. Jiang, Physics Letters B 659, 888 (2008), ISSN 0370-2693.

[11] J.R. and Mureika, Physics Letters B 660, 561 (2008), ISSN 0370-2693.

[12] J. McDonald, Journal of Cosmology and Astroparticle Physics 2009, 019 (2009).

[13] O. Bertolami, and P. Santos, Phys. Rev. D 79, 127702 (2009). 